\title{
Stochastic Programs and Hybrid Automata for (Biological) Modeling
}

\author{
Luca Bortolussi ${ }^{1}$ and Alberto Policriti ${ }^{2,3}$ \\ 1 Dept. of Mathematics and Informatics, University of Trieste, Italy. \\ 2 Dept. of Mathematics and Informatics, University of Udine, Italy. \\ 3 Istituto di Genomica Applicata, Udine, Italy.
}

\begin{abstract}
We present a technique to associate to stochastic programs written in stochastic Concurrent Constraint Programming a semantics in terms of a lattice of hybrid automata. The aim of this construction is to provide a framework to approximate the stochastic behavior by a mixed discrete/continuous dynamics with a variable degree of discreteness.
\end{abstract}

\section{Introduction}

In this paper we perform a further step in a program whose high-level aim is that of developing methods to replace stochastic approaches in (biological) modeling, by exploiting techniques for modeling a mixed (hybrid) continuous/discrete behavior. In other words, what we are exploring is the possibility of systematically eliminating the "stochastic ingredient", by the restriction to a discrete domain of some of the system's variables.

Our approach has been introduced and discussed in a sequence of papers (see(BP07; BP08c; $\mathrm{BP} 08 \mathrm{~b})$ ) and it is based on the following assumptions:

- there are situations in which stochastic models are - sometimes much-more realistic/precise;

- reducing the amount of the "stochastic ingredient" (however introduced) in a model, highly reduces the computational costs;

- in order to state precisely the scope and potential of a proposal like ours, two formalisms that will play the role of stochastic and hybrid reference languages, respectively, must be clearly fixed a-priori.

As far as the last point is concerned, our choices for the two formalisms have been the following:

1. Stochastic Concurrent Constraint Programming (sCCP);

2. Hybrid Automata (HA).

The above choices are, clearly, not the only possible ones and an extension of our proposal(s) to other pairs of languages, for the respective roles, is certainly possible. However, we believe such an extension is a non-trivial one, as the technicalities involved touch delicate aspects of the entire program: a language change does not seem to be a "syntactic sugar substitution". More on this point throughout the paper.

A general method to pass from a model written in Stochastic Concurrent Constraint Programming to a Hybrid Automata, has been introduced and discussed in (BP08a) and will be reviewed here. The Hybrid Automaton obtained is observed, in interesting cases, to retain significant properties of the stochastic simulator. Moreover, it is also shown how the level of discreteness introduced is an important parameter for evaluating the overall results of the approach. As an example, we mention the stable oscillations guaranteed for non-stochastic hybrid model of circadian clock, proposed in (BP08b) and obtained as counterpart of a $\mathbf{~ C C P}$-based model.

The specific contribution we put forward here is exploring the possibility of generalizing and deepening the above line of research, by trying to answer the following question: is it possible to control the amount of discreteness characterizing the HA proposed to simulate a given $\mathbf{~ C C P ~ p r o g r a m ? ~}$ 
We tackled the above problem with a refinement of our original proposal. We defined a mapping for sCCP-programs that instead of having a single HA as target, is designed to produce a lattice of HA's, whose level of discreteness determines the proximity to the top/bottom of the lattice.

As with previous experiences within our program, we had to begin from apparently secondary problems - e.g. a "massage" to the classical definition of HA - that, instead, turned out to address rather deep points. For example, the definition of Transition Driven Hybrid Automata we put forward, even though natural (discussed in some detail in Section (2)) was crucial for the successful completion of our task, as was addressing the basic problem of assigning a discrete state to the HA while the system is moving along a continuous path. We solved this problem by a mechanism assigning a tendency to be in a state among the possible ones, reminiscent of quantum-mechanics. Such a tendency must then be used to decode from transition's guard (our computational counterpart) the probabilistic choices to be made.

It can be argued that the stochastic dimension plays, in Systems Biology, a rather important role that can be seen as a way to render in-silico unknown - or computationally too complex-biological processes. This is one of the reasons for which we are developing our program in the context of Systems Biology, the other being that the kind of computation carried out by biological systems is the paradigmatic example of hybrid (discrete/continuous) computation.

\section{Hybrid Automata}

In this section we briefly recall the ideas and the definition of hybrid automata (HA). The reader is referred to (Hen96) for an introductory survey. Hybrid automata are dynamical systems presenting both discrete and continuous evolution. Essentially, they are defined using a set of variables evolving continuously in time, subject to instantaneous changes induced by the happening of discrete control events. When discrete events happen the automaton enters its next mode, where the laws governing the flow of continuous variables may change. Formally, a hybrid automaton can be defined as a tuple $\mathcal{H}=(V, E, \mathbf{X}$, flow, init, inv, jump, reset $)$, where:

$-\mathbf{X}=\left\{X_{1}, \ldots, X_{n}\right\}$ is a finite set of real-valued variables ${ }^{4}$.

$-G=(V, E)$ is a finite labeled multi-graph, called control graph. Vertices $v \in V$ are the (control) modes, while edges $e \in E$ are called (control) switches and model the happening of a discrete event.

- Associated with each vertex $v \in V$ there is a set of ordinary differential equations ${ }^{5} \dot{\mathbf{X}}=\operatorname{flow}(v)$ (referred to as the flow conditions). Moreover, $\operatorname{init}(v)$ and $\operatorname{inv}(v)$ are two formulae on $\mathbf{X}$ specifying the admissible initial conditions and some invariant conditions that must be true during the continuous evolution of variables in $v$ (forcing a change of mode to happen when violated).

- Each edge $e \in E$ of the control graph are labeled by $j u m p(e)$, a formula in $\mathbf{X}$ stating for what variables' values the transition is active (the corresponding region is called activation region), and by reset(e), a formula in $\mathbf{X} \cup \mathbf{X}^{\prime}$ specifying the change of the variables' values after the transition has taken place.

A valuation for the system's variables is denoted by $\mathbf{x}=\left(x_{1}, \ldots, x_{n}\right)$ and it is identified with a point in $\mathbb{R}^{n}$. The traces of the system are defined as the time traces of the continuous variables. Notice that the activation conditions are in general non-deterministic (as well as resets), hence there can be different traces starting from the same initial values. In this paper we are concerned mainly with simulation of hybrid automata, i.e. with the generation of a set of admissible traces.

\footnotetext{
${ }^{4}$ Notation: the time derivative of $X_{j}$ is denoted by $\dot{X}_{j}$, while the value of $X_{j}$ after a change of mode is indicated by $X_{j}^{\prime}$

${ }^{5}$ Other forms of flow specification are possible (differential inclusions, first order formulae, etc.) but sets of differential equations are sufficient for our purposes here.
} 


\subsection{Transition-driven Hybrid Automata}

In the previous section we introduced the classical formalization of hybrid automata. For the discussion to come, however, we found more convenient to use a different formalization of HA, which we will call Transition-Driven Hybrid Automata (TDHA). They can be quite straightforwardly mapped into standard ones, the basic idea in their definition being to prepare for a manipulation (a.k.a. merge/split) of discrete and continuous states. We focus on transitions, which can be either discrete (corresponding to the "standard" edges of HA) or continuous (representing flows acting on system's variables). At this stage, for the sake of definiteness, we flag as urgent (i.e. to be taken as soon as activated) all discrete transitions: just a matter of convenience, or rather an artefact of our interest in simulation of HA. Managing standard non-deterministic guards is, however, simply a matter of slightly modifying the definition of the standard HA associated to a TDHA. Formally:

Definition 1. A Transition-Driven Hybrid Automaton is a tuple $\mathcal{T}=(Q, \mathbf{X}$, inv, $\mathfrak{T} \mathfrak{C}, \mathfrak{T} \mathfrak{D}$, init $)$, where:

- $Q$ is a finite set of control modes, $\mathbf{X}=\left\{X_{1}, \ldots, X_{n}\right\}$ is a set of real valued system's variables, and for each $u \in Q$, inv $[u]$ is a quantifier-free first-order formula with free variables among $\mathbf{X}$, denoting the ivariant of $u$.

- $\mathfrak{T C}$ is the set of continuous transitions or flows, whose elements $\tau$ are triples (u, stoich, rate), where: $u \in Q$ is a mode, stoich is a vector of size $|\mathbf{X}|$, and rate $: \mathcal{X}_{u} \rightarrow \mathbb{R}$ is a function. The elements of a triple $\tau$ are indicated by $\operatorname{cmode}[\tau], \operatorname{stoich}[\tau]$, and rate $[\tau]$, respectively.

- TD is the set of discrete or instantaneous transitions, whose elements $\delta$ are quadruples of the form ( $u, v$,guard,reset), where: $u$ is the exit-mode, $v$ is the enter-mode, guard is a quantifier-free firstorder formula with free variables among $\mathbf{X}$, and reset is a quantifier-free first-order formula over $\mathbf{X}, \mathbf{X}^{\prime}$. The elements of a quadruple $\delta$ are indicated by $\operatorname{exit}[\delta]$, enter $[\delta]$, guard $[\delta]$, and $\operatorname{reset}[\delta]$, respectively.

- For each $u \in Q$, init $[u]$ is a quantifier-free first-order formula over $\mathbf{X}$, defining the initial conditions of the system.

For each $u \in Q$ he formula $i n v[u]$ characterizes the set of admissible values that can be taken by system's variables while in mode $u$. We will also denote by $\mathcal{X}_{u}$ the set $\mathcal{X}_{u}=\{\mathbf{x} \mid \operatorname{inv}[u, \mathbf{x}]\}$ - the states space of mode $u$ - and by $\mathcal{X}=\bigcup_{u \in Q}\{u\} \times \mathcal{X}_{u}$ the hybrid states space.

For each $\tau \in \mathfrak{T C}, \operatorname{stoich}[\tau]$ and rate $[\tau]$ give the magnitude and the form of the action of $\tau$ on each variable $X \in \mathbf{X}$, respectively (see below).

For each $\delta \in \mathfrak{T} \mathfrak{D}, \operatorname{guard}[\delta]$ represents the region of $\mathcal{X}_{u}$ in which the transition $\delta$ is active and reset $[\delta]$ expresses the relation intervening among variables $(\mathbf{X})$ - before the transition - and $\left(\mathbf{X}^{\prime}\right)$ after.

The spirit behind the above definition is quite simple. We explicitly define automaton's modes introducing two kind of edges. Continuous edges self-loop on a mode and represent a flow acting locally on that mode. Their mathematical form is given by the rate, while their effect on system's variables is given by the stoichiometry vector. Discrete edges, instead, connect two modes (they can also self-loop), are active only in specific regions of the state space (identified by their guards), and can change the value of some variables when executed (as predicated by their reset). TDHA can be encoded quite simply into classical hybrid automata following the prescriptions of the subsequent definition.

Definition 2. Let $\mathcal{T}=(Q, \mathbf{X}$, inv, $\mathfrak{T} \mathfrak{C}, \mathfrak{T} \mathfrak{D}$, init $)$ be a transition-driven hybrid automaton. The hybrid automaton $\mathcal{H}_{\mathcal{T}}=\left(V, E, \mathbf{X}\right.$, flow, init $_{\mathcal{H}}$, inv $_{\mathcal{H}}$, jump, reset $)$ associated to TDHA is given by:

1. the set of modes $V$ is $Q$;

2. the initial conditions init $_{\mathcal{H}}$ are init;

3. for each $v \in V$, inv $v_{\mathcal{H}}[v] i s, i n v[v] \wedge\left(\bigwedge_{\delta \in \mathfrak{T} \mathfrak{D} \mid \operatorname{exit}[\delta]=v} \neg \operatorname{guard}[\delta]\right)$;

4. flow $[v]: \mathcal{X}_{v} \rightarrow \mathbb{R}^{n}$, is defined as flow $[v](\mathbf{X})=\sum_{\tau \in \mathfrak{T C} \mid \operatorname{cmode}[\tau]=v} \operatorname{stoich}[\tau] \cdot \operatorname{rate}[\tau]$;

5. the control edges are the set $E=\{(\operatorname{exit}[\delta]$, enter $[\delta]) \mid \delta \in \mathfrak{T} \mathfrak{D}\}$; 
6. guards and resets for each edge $e \in E$ where $e=(\operatorname{exit}[\delta]$, enter $[\delta])$, are simply $\operatorname{guard}[e]=\operatorname{guard}[\delta]$ and $\operatorname{reset}[e]=\operatorname{reset}[\delta]$.

The only non-trivial part of the above definition is the flows construction from the set $\mathfrak{T C}$ of continuous transitions. Essentially, for each transition $\tau \in \mathfrak{T C}$ active in a mode $v$, we multiply the function $\operatorname{rate}[\tau](\mathbf{X})$ for the magnitude $\operatorname{stoich}[\tau][X]$ for each variable $X \in \mathbf{X}$ and add these terms to obtain the ODE's. For instance, consider a system with two variables $X$ and $Y$, a single mode $u$, and the following set $\mathfrak{T C}=\left\{\left(u,\langle 0,1\rangle, Y^{2}\right),\left(u,\langle 1,0\rangle, X^{2}\right),(u,\langle-1,-1\rangle, X \cdot Y)\right\}$. Then the ODE for $X$ is flow $[u, X]=0 \cdot Y^{2}+1 \cdot X^{2}+(-1) \cdot X Y=X^{2}-X Y$, while for $Y$ we get flow $[u, Y]=Y^{2}-X Y$. Notice also that the definition of the invariant conditions being the formula $\bigwedge_{\delta \in \mathfrak{T} \mathfrak{D} \mid \operatorname{exit}[\delta]=v} \neg \operatorname{guard}[\delta]$ — which is true when no discrete transition is active - forces the system to leave a mode whenever a transition becomes true. This is in agreement with the urgent semantics of discrete transitions of TDHA.

\subsection{Product of TDHA}

We will define now a notion of product for TDHA. As a preliminary notion, we extend the embedding of states into pairs of states to continuous and discrete transitions. Let $\mathcal{T}=\left(Q, \mathbf{X}\right.$, inv $_{1}, \mathfrak{T C}, \mathfrak{T} \mathfrak{D}$, init $\left._{1}\right)$ be a TDHA, $\tau \in \mathfrak{T C}$ be a continuous transition, $u$ be a mode in a set $Q^{\prime}, Q^{\prime} \cap Q=\emptyset$, and $\mathbf{Y}$ be a set of variables, with $\mathbf{X} \subseteq \mathbf{Y}$. The embedding $\mathfrak{i}_{1}$ of $\tau$, w.r.t. $u$ and $\mathbf{Y}$ is defined as $\mathfrak{i}_{1}(\tau, u, \mathbf{Y})=$ $\left((\operatorname{comode}[\tau], u), \operatorname{stoich}[\tau]^{\mathbf{Y}}, \operatorname{rate}[\tau]\right)$, with $\operatorname{stoich}[\tau]^{\mathbf{Y}}$ a vector on $\mathbf{Y}$ coinciding with $\operatorname{stoich}[\tau]$ for each $X \in \mathbf{X}$, and equal to zero for $X \notin \mathbf{X}$. Similarly $\mathfrak{i}_{2}(\tau, u, \mathbf{Y})$ is defined as $\left((u, \operatorname{cmode}[\tau]), \operatorname{stoich}[\tau]^{\mathbf{Y}}, \operatorname{rate}[\tau]\right)$. An analogous notion of embedding can be defined for discrete transitions: for $\delta \in \mathfrak{T} \mathfrak{D}$ we set

$$
\mathfrak{i}_{1}(\delta, u, \mathbf{Y})=((\operatorname{exit}[\delta], u),(\operatorname{enter}[\delta], u), \operatorname{guard}[\delta], \operatorname{reset}[\delta])
$$

and

$$
\mathfrak{i}_{2}(\delta, v, \mathbf{Y})=((v, \operatorname{exit}[\delta]),(v, \operatorname{enter}[\delta]), \operatorname{guard}[\delta], \operatorname{reset}[\delta]) .
$$

Definition 3. Given two TDHA $\mathcal{T}_{i}=\left(Q_{i}, \mathbf{X}_{\mathbf{i}}\right.$, inv $_{1}, \mathfrak{T C}_{i}, \mathfrak{T D}_{i}$, init $\left._{1}\right)$ for $i \in\{1,2\}$, their product $\mathcal{T}=$ $\mathcal{T}_{1} \otimes \mathcal{T}_{2}, \mathcal{T}=\left(Q, \mathbf{X}\right.$, inv $_{1}, \mathfrak{T} \mathfrak{C}, \mathfrak{T} \mathfrak{D}$, init $\left._{1}\right)$, is defined by:

$-Q=Q_{1} \times Q_{2}, \mathbf{X}=\mathbf{X}_{\mathbf{1}} \cup \mathbf{X}_{\mathbf{2}}$, and $i n v[(u, v)]=\operatorname{inv}_{1}[u] \wedge i n v_{2}[v]$

$-\mathfrak{T C}=\left\{\mathfrak{i}_{1}(\tau, v, \mathbf{X}) \mid \tau \in \mathfrak{T C}_{1}, v \in Q_{2}\right\} \cup\left\{\mathfrak{i}_{2}(\tau, u, \mathbf{X}) \mid \tau \in \mathfrak{T C}_{2}, u \in Q_{1}\right\} ;$

$-\mathfrak{T D}=\left\{\mathfrak{i}_{1}(\delta, v, \mathbf{X}) \mid \delta \in \mathfrak{T D}_{1}, v \in Q_{2}\right\} \cup\left\{\mathfrak{i}_{2}(\delta, u, \mathbf{X}) \mid \delta \in \mathfrak{T D}_{2}, u \in Q_{1}\right\} ;$

$-\operatorname{init}[(u, v)]=$ init $_{1}[u] \wedge$ init $_{2}[v]$.

The definition is quite natural, given the absence of synchronization mechanisms for TDHA, yet we wish to focus the attention of the reader on the effect on flows. In fact, for each variable shared between $\mathcal{T}_{1}$ and $\mathcal{T}_{2}$, the result obtained is the addition of the right-hand sides of the corresponding differential equations. Hence, this construction is equivalent to the flux product defined in (BP08a).

\section{Stochastic Concurrent Constraint Programming}

In this section we present the (computational/programming) language we chose to introduce the stochastic dimension in our picture. This language will be used as source for the mapping into the continuous/discrete language of HA's, mentioned in the introduction. Many possible choice for introducing a stochastic ingredient in a programming language were possible and we decided to use (a simplified version of) stochastic Concurrent Constraint Programming (sCCP (Bor06), a stochastic extension of CCP (Sar93)), as it seems to be sufficiently expressive, compact, and especially suitable in many other respects - to be discussed-, for our purposes ${ }^{6}$.

\footnotetext{
${ }^{6}$ There are other probabilistic extensions of CCP studied in literature, like (DW98; GJS97; GJP99). (DW98) provides CCP with a semantics based on discrete time Markov Chains, while in (GJS97; GJP99) the stochastic ingredient is introduced by extending the store with random variables and adding a primitive for sampling. These approaches, however, are not suited for our purposes, as we need a model in which events happen probabilistically in continuous-time, as customary in biochemical modeling.
} 
A (simplified) sCCP program consists of a set of agents, whose syntactic and semantic nature will be discussed shortly, interacting via a collection of formulae recorded as a shared store. The interaction initially takes place through concurrent modification of a finite set of variables $\mathbf{X}=\left\{X_{1}, \ldots, X_{n}\right\}-$ usually taking integer values, even though more structured numerical domains are possible - and continues via logical entailment defined on formulae occurring in the shared store. It is sufficient to define a configuration $\mathbf{c}$ of the store simply as a valuation of the variables $\mathbf{X}$ and the basic action $\pi$ executable by agents as a guarded update of (some of the) variables: $g(\mathbf{X}) \rightarrow u\left(\mathbf{X}, \mathbf{X}^{\prime}\right)$. In a guarded update $g(\mathbf{X})$ is a propositional formula whose atoms are inequality predicates on variables $\mathbf{X}$ and $u\left(\mathbf{X}, \mathbf{X}^{\prime}\right)$ is a conjunction of predicates on $\mathbf{X}, \mathbf{X}^{\prime}$ of the form $\mathbf{X}^{\prime}=\mathbf{f}(\mathbf{X})$ ( $\mathbf{X}^{\prime}$ denotes variables of $\mathbf{X}$ after the update), for some function $\mathbf{f}: \mathbb{R}^{n} \rightarrow \mathbb{R}^{n}$. The notion of entailment of CCP (see (Sar93)) takes here a very simple form: a guard $g(\mathbf{X})$ is entailed by a configuration $\mathbf{c}, \mathbf{c} \vdash g(\mathbf{X})$, simply if $g(\mathbf{c})$ is true. If $\mathbf{f}(\mathbf{X})=\mathbf{X}+\mathbf{k}$, with $\mathbf{k}$ a vector of constants, we talk of constant updates. A full-fledged use of entailment and more complicated update functions would empower the language but are not necessary for illustrating the technique at the focus of this paper.

In addition, the language has all the basic constructs of process algebras: non-deterministic choice, parallel composition, and recursive calls. The characteristic feature of stochastic CCP is the fact that each action $\pi$ is given a stochastic duration, specified by associating an exponentially distributed random variable to actions, whose rate depends on the state of the system through a function $\lambda: \mathbf{X} \rightarrow$ $\mathbb{R}^{+}$. Stochastic actions are denoted by $[\pi]_{\lambda}$ and the possibility of having $\lambda$ dependent from the entire state of the system (at least as far as it is recognizable by the system's variable values) is one of the convenient features motivating the choice of SCCP.

Definition 4. A $s \boldsymbol{C C P}$ program is a tuple $\mathcal{A}=(A, \mathcal{D}, \mathbf{X}$, dom, init $(\mathbf{X}))$, where

1. The initial agent $A$ and the set of definitions $\mathcal{D}$ are given by the following grammar

$$
\begin{aligned}
\mathcal{D} & =\emptyset|\mathcal{D} \cup \mathcal{D}|\{C \stackrel{\text { def }}{=} M\} \\
\pi & =\left[g(\mathbf{X}) \rightarrow u\left(\mathbf{X}, \mathbf{X}^{\prime}\right)\right]_{\lambda(\mathbf{X})} \\
M & =\pi . C \mid M+M \\
A & =M \mid A \| A
\end{aligned}
$$

2. $\mathbf{X}$ is the set of variables of the store (with global scope);

3. dom is a formula with free variables in $\mathbf{X}$, specifying the domain of each variable.

4. init $(\mathbf{X})$ is a predicate on $\mathbf{X}$ of the form $\mathbf{X}=\mathbf{x}_{\mathbf{0}}$, assigning an initial value to store variables.

The variables used in the store are assumed to have global scope and dom is a conjunction of quantifier-free formulae on a single variable $X$, with atoms consisting of equality and inequality predicates. The conjunct for variable $X$ is identified by $\operatorname{dom}[X]$. An example is given in Appendix (A).

Operational semantics. The operational semantics of SCCP is given by a transition relation that generates a Labeled Transition System (LTS), from which a Continuous Time Markov Chain (CTMC (Nor97)) can be inferred, see (BP08c; Bor06) for further details. We briefly sketch the mechanism:

- A congruence relation $\equiv$ is defined on the set of all possible agents, whose collection of equivalence classes is indicated by $\mathfrak{A}$. On $\mathfrak{A}$ the algebraic structure of a monoid is induced by stochastic summation + and parallel composition $\|$.

- A configuration of the system is a pair $\langle A, \mathbf{x}\rangle \in \mathfrak{A} \times \mathbb{R}^{n}$, with $A$ a parallel agent (modulo $\equiv$ ) and $\mathbf{x} \in \mathbb{R}^{n}$ a valuation of the system's variables.

- The transition relation $\longrightarrow \subseteq\left(\mathfrak{A} \times \mathbb{R}^{n}\right) \times \mathbb{R}^{+} \times\left(\mathfrak{A} \times \mathbb{R}^{n}\right)$, defining the LTS-details are given in Appendix (B) . The labels of the transition relation record the actual rates of the transitions.

- From the LTS we can obtain a Continuous Time Markov Chain (CTMC), described through the infinitesimal generator matrix (cf. (Nor97)), as follows: the rate on the edge connecting states $s_{1}$ and $s_{2}$ is obtained summing the rates of all LTS-edges between $s_{1}$ and $s_{2}$. 
All agents definable in $\mathbf{s C C P}$, i.e. all agents $C \stackrel{\text { def }}{=} M \in \mathcal{D},{ }^{7}$ are sequential, i.e. they do not contain any occurrence of the parallel operator, whose usage is restricted at the upper level of the network. Indeed, a sCCP program is the parallel composition of sequential agents. Moreover, all variables are global, hence no parameter is passed in recursive calls.

sCCP sequential agents can be seen as automata synchronizing on store variables and they can be conveniently represented as labeled graphs, called Reduced Transition Systems (RTS) in (BP07). A formal definition is possible and is fully specified in Appendix (B). The steps to obtain an object suitable for the following treatment are:

1. define the collection of all possible states - the derivative set $\operatorname{Der}(C)$ - and actions - action $(C)$ of any sequential agent appearing in a $\mathbf{~ S C C P}$ simple program (i.e. without multiple copies of the same agent) $\mathcal{A}=(A, \mathcal{D}, \mathbf{X}, \operatorname{dom}$, init $(\mathbf{X}))$;

2. on the ground of the above definition the following LTS multi-graph $R T S(C)=(S(C), E(C), \ell)$ is introduced:

$-S(C)=\operatorname{Der}(C)$,

- $E(C)=\{(\operatorname{exit}(\pi)$, enter $(\pi)) \mid \pi \in \operatorname{action}(C)\}$,

- $\ell(e)=(\operatorname{guard}(\pi)$, update $(\pi), \operatorname{rate}(\pi))$, where $\pi$ is the action defining the edge $e \in E(C)$;

3. the notion of extended $\mathbf{~} \mathbf{C C P}$ program $\mathcal{A}^{+}=\left(A^{+}, \mathcal{D}^{+}, \mathbf{Y}\right.$, dom $^{+}$, init $\left.^{+}(\mathbf{Y})\right)$, in which a counter for run-time recording the number of parallel copies of each agent, is introduced and proved isomorphic to $\mathcal{A}$. Essentially, this is a technical trick that simplifies the overall treatment: we introduce a new variable $P_{C}$ for each component $C \in \mathcal{D}$ of a $\mathbf{S C C P}$ program. These variables will count the number of copies of $C$ present in parallel in the system at a certain time. To take into account the effects of transitions on agents, we modify updates and rate functions.

Definition 5. Let $\mathcal{A}=(A, \mathcal{D}, \mathbf{X}, \operatorname{dom}$, init $(\mathbf{X}))$ be a sCCP program. The extension $\mathcal{A}^{+}=\left(A^{+}, \mathcal{D}^{+}, \mathbf{Y}\right.$, dom $^{+}$, init $\left.^{+}(\mathbf{Y})\right)$ of $\mathcal{A}$ is defined by:

$-\mathbf{Y}=\mathbf{X} \cup \mathbf{P}$, where $\mathbf{P}=\left\{P_{C} \mid C \in \mathcal{D}\right\}$.

- Each $C \stackrel{\text { def }}{=} M \in \mathcal{D}$, with $M=\sum_{i=1}^{k} \pi_{i} . C_{i}$, is replaced by $C^{+} \stackrel{\text { def }}{=} M^{+}, M^{+}=\sum_{i=1}^{k} \pi_{i}^{+} . C_{i}^{+}$.

- If $\pi_{i}=\left[g_{i}(\mathbf{X}) \rightarrow u_{i}\left(\mathbf{X}, \mathbf{X}^{\prime}\right)\right]_{\lambda_{i}(\mathbf{X})}$, then $\pi_{i}^{+}=\left[g_{i}(\mathbf{X}) \rightarrow u_{i}\left(\mathbf{Y}, \mathbf{Y}^{\prime}\right)^{+}\right]_{\lambda_{i}^{+}(\mathbf{Y})}$, where $\lambda_{i}^{+}(\mathbf{Y})=$ $P_{C} \cdot \lambda_{i}(\mathbf{X})$ and $u_{i}\left(\mathbf{Y}, \mathbf{Y}^{\prime}\right)^{+}=u_{i}\left(\mathbf{X}, \mathbf{X}^{\prime}\right) \wedge P_{C}^{\prime}=P_{C}-1 \wedge P_{C_{i}}^{\prime}=P_{C_{i}}+1$ if $C \neq C_{i}, u_{i}\left(\mathbf{Y}, \mathbf{Y}^{\prime}\right)^{+}=$ $u_{i}\left(\mathbf{X}, \mathbf{X}^{\prime}\right)$ otherwise.

- If $A=C_{1}\|\ldots\| C_{n}$, then $A^{+}=C_{1}^{+}\|\ldots\| C_{n}^{+}$

$-\operatorname{dom}^{+}=\operatorname{dom} \wedge\left(\bigwedge_{C \in \mathcal{D}} P_{C} \geq 0\right)$

- $\operatorname{init}^{+}(\mathbf{Y})=\operatorname{init}(\mathbf{X}) \wedge \bigwedge_{C \in \mathcal{D}}\left(P_{C}=\#(C, A)\right)$, with \#(C,A) denoting the number of copies of $C$ in $A$.

On the ground of the following proposition (whose proof can be found in Appendix (B)) we have that the extension of a simple sCCP program does not alter the CTMC obtained.

Proposition 1. Let $\mathcal{A}$ be a simple sCCP program and $\mathcal{A}^{+}$be its extension. The LTS of $\mathcal{A}$ and $\mathcal{A}^{+}$are isomorphic as labeled multi-graphs.

Hence, for a simple sCCP programs $\mathcal{A}$ we can work indistinguishably with $\mathcal{A}$ or with its extension $\mathcal{A}^{+}$. This property is no more true for non-simple agents, as $P_{C}$ can take values greater than one, hence rate $[\pi]$ is generally different from rate $\left[\pi^{+}\right]$.

Remark 1. State variables in simple program may seem useless: they can only take values 0 or 1. We will see in the next section that, instead, their continuous approximation in the interval $[0,1]$ will provide the mechanism for controlling a cluster of discrete states (continuously approximated). Basically, the real value of a state variable will indicate the "tendency" of the system to be in that particular state. The sum of such tendencies for discrete states merged in a continuous cluster, will always be constrained to be equal to 1 .

\footnotetext{
${ }^{7}$ In the following, with a slight abuse of notation, we sometimes write $C \in \mathcal{D}$ for $C \stackrel{\text { def }}{=} M \in \mathcal{D}$.
} 
At this point we can employ a method to turn the effect of each sequential agent state's variables into a suitable mathematical form. Following (BP07) we can define a fluid-flow approximation of sCCP. The method works by approximating as continuous the variables $\mathbf{X}$ and the state variables $\mathbf{P}$, and considering the effect of each transition on those variables. To this end two additional restrictions on SCCP programs are required: all updates must be of the constant type and the program must be simple (cf. Definition 12). The method proceeds along the following steps:

1. Start from a simple $\mathbf{~} \mathbf{C C P}$ program $\mathcal{A}=(A, \mathcal{D}, \mathbf{X}, \operatorname{dom}$, init $(\mathbf{X}))$, extend it to construct $\mathcal{A}^{+}=$ $\left(A^{+}, \mathcal{D}^{+}, \mathbf{Y}\right.$, dom $^{+}$, init $\left.^{+}(\mathbf{Y})\right)$ and build the RTS for each component $C$ occurring in parallel in $A^{+}$.

2. For each $R T S(C)$, define the interaction matrix $\nu_{C}$ as a matrix with rows indexed by $\mathbf{Y}$ and columns indexed by edges $e \in E(C)$. The entry $\nu_{C}[X, e]$ contains the net variation on $X$ induced by update (e) (for instance, if $X^{\prime}=X+2$ is the (only) conjunct of update $(e)$ on $X$, then $\nu_{C}[X, e]=$ $2)$. In addition, we consider also the rate vector $\phi_{C}$, indexed by edges $e \in E(C)$, defined by $\phi_{C}[e]=\operatorname{rate}(e) \cdot I(\operatorname{guard}(e))$, where $I(\cdot)$ is the indicator function.

3. The differential equations for agent $C$ are $\dot{\mathbf{Y}}=\Phi_{C}(\mathbf{Y})$, with $\Phi_{C}=\nu_{C} \cdot \phi_{C}$. If $A=C_{1}\|\ldots\| C_{n}$, then the differential equations for $A$ are $\dot{\mathbf{Y}}=\Phi_{A}(\mathbf{Y})=\Phi_{C_{1}}(\mathbf{Y})+\ldots+\Phi_{C_{n}}(\mathbf{Y})$.

\section{From sCCP to TDHA}

In this section we touch the core of our proposal, specifying our mapping in the case of a sCCP program associated to a Transition-Driven Hybrid Automaton, in such a way to deal with a lattice of different TDHA's.

First of all recall that transitions in $\mathbf{S C C P}$ are all discrete and stochastic. The idea behind the translation is simply to approximate some transition as continuous, leaving the others discrete. Stochasticity is mimicked by the introduction of suitable timing conditions for the discrete transitions.

The mapping proceeds in two steps. First we convert into TDHA's each sequential component of a sCCP program, then all these TDHA's are composed together using the product construction of Section (2.2).

Given a $\mathcal{A}^{+}=\left(A^{+}, \mathcal{D}^{+}, \mathbf{Y}\right.$, dom $^{+}$, init $\left.^{+}(\mathbf{Y})\right)$, let $C \in \mathcal{D}^{+}$be an agent in parallel in the initial agent $A$ and let $R T S(C)=(S(C), E(C), \ell)$ be its RTS. The basic idea of the mapping is to partition the set of transitions $E(C)$ according to a choice of a specific continuous/discrete scheme of approximation. In order to formalize this, we use a boolean vector $\kappa \in\{0,1\}^{m}, m=|E(C)|$, indexed by edges in $E(C)$ : for $e \in E(C), \kappa[e]=1$ means that the transition will be approximated as continuous, while $\kappa[e]=0$ implies that it will remain discrete. Technically, we partition $E(C)$ in two disjoint subsets: $E(\kappa, C)=$ $\{e \in E(C) \mid \kappa[e]=1\}$ (to be approximated as continuous) and $E(\neg \kappa, C)=\{e \in E(C) \mid \kappa[e]=0\}$ (to be approximated as discrete).

We need the following restriction to guarantee the existence of solutions to ODE's that will be introduced subsequently:

Definition 6. A s CCP action $\pi$ is called continuously approximable iff rate $(\pi)(\mathbf{X})$ is differentiable and $\operatorname{rate}(\pi)(\mathbf{X})=0$ whenever guard $(\pi)(\mathbf{X})$ is false.

Hence, for continuously approximable actions guards are, essentially, irrelevant. In fact, when a guard is false the corresponding transition is already deadlocked due to its rate having value zero (to see this is sufficient to check Table 1 in Appendix (B): having a rate greater than zero is a precondition on the transition). We call consistent a vector $\kappa$ which is equal to $1, \kappa[e]=1$, only for edges $e$ that are continuously approximable. In the following, we suppose to work only with consistent $\kappa$.

At this point we are ready to introduce the basic components of our target TDHA. 
Discrete Modes. The modes of the TDHA will be, as first approximation, essentially the states $S(C)$ of $R T S(C)$. However, clearly, since continuous transitions cannot change mode, we need to consider as equivalent those states that can be reached by a path of edges that $\kappa$ indicates to maintain continuous ${ }^{8}$.

Definition 7. Let $R T S(C)$ be the RTS of agent $C$, let $E(\kappa, C) \subseteq E(C)$ be the subset of continuous transitions, and let $s_{1}, s_{2} \in S(C) . s_{1} \sim_{\kappa} s_{2}$ iff there is a path of edges in $E(\kappa, C)$ from $s_{1}$ to $s_{2}$ in the non-oriented version of $R T S(C)$.

Clearly $\sim_{\kappa}$, being a reachability relation in a non-oriented graph, is an equivalence relation. Hence we can define the set $S_{\kappa}(C)=S(C) / \sim_{\kappa}$ of RTS-states modulo $\sim_{\kappa}$. For each state $[s] \in S_{\kappa}(C)$, we define the following objects:

- The local interaction matrix $\nu_{\mathbf{Y}, E(\kappa, C,[s])}$ w.r.t. variables $\mathbf{Y}$ and continuous edges $E(\kappa, C,[s])=$ $\left\{e=\left(s_{1}, s_{2}\right) \in E(\kappa, C) \mid s_{1}, s_{2} \in[s]\right\}$, that is edges self-looping on $[s] . \nu_{\mathbf{Y}, E(\kappa, C,[s])}$ has rows indexed by variables in $\mathbf{Y}$ and columns indexed by transitions in $E(\kappa, C,[s]): \nu_{\mathbf{Y}, E(\kappa, C,[s])}[X, e]=h$ if and only if variable $X$ is updated by transition $e$ according to the formula $X^{\prime}=X+h$.

- The local rate vector $\phi_{E(\kappa, C,[s])}$ w.r.t. transitions in $E(\kappa, C,[s])$ has $|E(\kappa, C,[s])|$ entries, and it is defined by $\phi_{E(\kappa, C,[s])}[e]=\operatorname{rate}(e)$.

Appendix (A) will illustrate the above notions.

Continuous flow. The continuous evolution for TDHA is given, in each mode, by the set $\mathfrak{T C}$ of continuous transitions defined as follows. To each edge $e \in E(\kappa, C,[s])$ looping on $[s]$ we associate the continuous transition

$$
\left([s], \nu_{\mathbf{Y}, E(\kappa, C,[s])}[\cdot, e], \phi_{E(\kappa, C,[s])}[e]\right),
$$

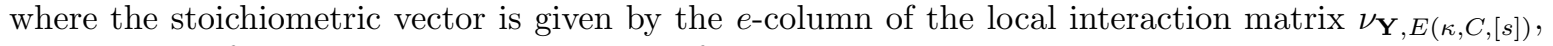
and the rate of the transition is the $e$-entry of the local rate vector $\phi_{E(\kappa, C,[s])}$.

Therefore, summarizing, (see Appendix (A)):

$$
\mathfrak{T C}=\left\{\left([\operatorname{exit}(e)], \nu_{\mathbf{Y}, E(\kappa, C,[s])}[\cdot, e], \phi_{E(\kappa, C,[s])}[e]\right) \mid e \in E(\kappa, C)\right\} .
$$

Invariant conditions. The space of admissible values is restricted by the domain constraints of the sCCP program. As we work with simple sCCP programs, we know that state variables $P_{C}$ always belong to the interval $[0,1]$. Hence, we can enforce the following invariant for each mode $v \in S_{\kappa}(C)$ : $\operatorname{inv}[v]=\operatorname{dom} \wedge\left(\bigwedge_{C^{\prime} \in \operatorname{Der}(C)} P_{C^{\prime}} \leq 1\right)$.

Discrete transitions. In order to deal with discrete transitions $e \in E(\neg \kappa, C)$, we need to take into account the timing of the corresponding events. As we need to remove the stochasticity, we fire the transition at its expected time. As some variables of the system evolve continuously, the stochastic transition becomes a non-homogeneous Poisson process (Ros96), with rate $\lambda(\mathbf{Y}(t))=\lambda(t)$. In this case, given the cumulative rate at time $t, \Lambda(t)=\int_{0}^{t} \lambda(\mathbf{Y}(s)) d s$, the probability of firing within time $t$ is $F(t)=1-e^{-\Lambda(t)}$. The random variable $T$ with this distribution can be simulated by an uniform random variable $U$ on $(0,1)$ solving for $t$ the equation $\Lambda(t)=-\log U$. Taking the average on both sides, we obtain $E[\Lambda(t)]=1$, hence we can fire the transition whenever $\Lambda(t) \geq 1 .{ }^{9}$ In order to describe this condition within the TDHA framework, we introduce a new variable $Z_{e}$, with initial value 0 , evolving deterministically according to the equation $\dot{Z}_{e}=\lambda(\mathbf{Y})$. The definition of this equation can be added to $\mathfrak{T C}$ in the obvious way, i.e. by adding the continuous edge ([exit $(e)], i d_{Z_{e}}, \lambda(\mathbf{Y})$ ), with $i d_{Z_{e}}$ the vector

${ }^{8}$ Actually, a distinction among those collapsed states will be maintained through the dependence on state variables $\mathbf{P}$, added in the extension $\mathcal{A}^{+}$. See Appendix (B).

$9 \Lambda(t)$ is a monotonic increasing function, due to non-negativity of $\lambda$, hence $\lambda(t)$ has a unique solution provided, for instance, $\Lambda(t) \rightarrow \infty$, a condition usually satisfied. If this does not happen, then there is a non-null probability that the transition never fires, hence the guard of the urgent transition may never become true. 
equal to one for the component associated to the variable $Z_{e}$ and zero elsewhere ${ }^{10}$. When the transition fires, we also reset all the variables $Z_{e}$ to zero, in order to mimic the memoryless property of CTMCs.

In the definition of discrete transitions, we also need to take into account guards and updates of the corresponding sCCP edge, the guard(e) and update(e) formulae, which will be part of guards and resets of the discrete transition. There are, however, two complications:

- It may happen that the reset on a variable, say $X$, brings its value out of the allowed domain. Such transitions should not be permitted: they can be avoided by adding suitable conditions to the guard. Specifically, if update $(e):=\mathbf{X}^{\prime}=\mathbf{f}(\mathbf{X}) \wedge \mathbf{P}^{\prime}=\mathbf{P}+\mathbf{h}$, we add to it the formula $\operatorname{dom}[\mathbf{X} / \mathbf{f}(\mathbf{X})]$ obtained by replacing each system's variable $X$ with the term $f_{X}(\mathbf{X})$ in the formula expressing domain constraints. This forces the values of $\mathbf{X}$ after the transition to satisfy domain constraints.

- The updates on state variables $\mathbf{P}$ reflect the change of states of the components. In particular, as the $\mathbf{S C C P}$ program is simple, they can assume just two values, 0 and 1. Actually, in the TDHA they can take values in the whole $[0,1]$ real interval, especially in the case in which we are currently in a clustered state $[s]$, collapsing states $s_{1}, \ldots, s_{n}$ of $R T S(C)$. In this case, the state variables $P_{s_{1}}, \ldots, P_{s_{n}}$ sum up to 1 , and generally they have values $0<P_{s_{i}}<1$, representing how likely is the $s_{i}$ state in the cluster $[s]$. In order to deal with those variables correctly, we need to insure that 1. when a state $[s]$ is left, all its state variables are set to zero and

2. when a discrete transition looping in $[s]$ happens, then the variable of its target state $s_{i}$ becomes equal to 1 , while all other variables of $[s]$ are reset to 0 .

We can obtain this in the following way. Consider an SCCP edge connecting states $s_{1}$ and $s_{2}$, with update update $(e):=\mathbf{X}^{\prime}=\mathbf{f}(\mathbf{X}) \wedge P_{s_{1}}^{\prime}=P_{s_{1}}-1 \wedge P_{s_{2}}^{\prime}=P_{s_{2}}+1$, and fix the vector $\kappa$. We will substitute the update of state variables $P_{s_{1}}^{\prime}=P_{s_{1}}-1 \wedge P_{s_{2}}^{\prime}=P_{s_{2}}+1$ by the formula update_statevars $(\mathbf{P})$, defined by

1. When $\left[s_{1}\right] \neq\left[s_{2}\right]$, update_statevars $(\mathbf{P})=\bigwedge_{s \in\left[s_{1}\right]} P_{s}^{\prime}=0 \wedge P_{s_{2}}^{\prime}=1$.

2. When $\left[s_{1}\right]=\left[s_{2}\right]$, update_statevars $(\mathbf{P})=\bigwedge_{s \in\left[s_{1}\right], s \neq s_{2}} P_{s}^{\prime}=0 \wedge P_{s_{2}}^{\prime}=1$.

Putting all this discussion together, we have that the discrete transition associated to $e \in E(\neg \kappa, C)$ with $e=\left(s_{1}, s_{2}\right)$ and update $(e):=\mathbf{X}^{\prime}=\mathbf{f}(\mathbf{X}) \wedge P_{s_{1}}^{\prime}=P_{s_{1}}-1 \wedge P_{s_{2}}^{\prime}=P_{s_{2}}+1$ is (see Appendix (A))

$$
\left(\left[s_{1}\right],\left[s_{2}\right], \operatorname{guard}(e) \wedge \operatorname{dom}[\mathbf{X} / \mathbf{f}(\mathbf{X})] \wedge Z_{e} \geq 1, \mathbf{X}^{\prime}=\mathbf{f}(\mathbf{X}) \wedge \text { update_statevars }(\mathbf{P}) \wedge\left(\bigwedge_{e \in E(C)} Z_{e}^{\prime}=0\right)\right) \in \mathfrak{T} \mathfrak{D} .
$$

We can now collect all our considerations into a global definition.

Definition 8. Let $\mathcal{A}=\left(A, \mathcal{D}, \mathbf{X}\right.$, dom, init $\left.t_{0}\right)$ be a simple $\boldsymbol{s} \boldsymbol{C C P}$ program and $\mathcal{A}^{+}=\left(A^{+}, \mathcal{D}^{+}, \mathbf{Y}\right.$, dom $^{+}$, init $\left._{0}^{+}\right)$ be its extended version. Let $C$ be a sequential component in parallel in $A^{+}$, with $R T S(C)=(S(C), E(C), \ell)$. Fix a boolean vector $\kappa \in\{0,1\}^{m}, m=|E(C)|$. The Transition-Driven Hybrid Automaton associated to $C$ w.r.t. $\kappa$ is $\mathcal{T}(C, \kappa)=(Q, \mathbf{Z}$, inv, $\mathfrak{T C}, \mathfrak{T} \mathfrak{D}$, init $)$, where

$-Q=S_{\kappa}(C)=S(C) / \sim_{\kappa}, \mathbf{Z}=\mathbf{Y} \cup\left\{Z_{e} \mid e \in E(C)\right\}$, and inv $[v]=\operatorname{dom} \wedge\left(\bigwedge_{C^{\prime} \in \operatorname{Der}(C)} P_{C^{\prime}} \leq 1\right)$;

$-\mathfrak{T C}=\left\{\left([\operatorname{exit}(e)], \nu_{\mathbf{Y}, E(\kappa, C,[s])}[\cdot, e], \phi_{E(\kappa, C,[s])}[e]\right) \mid e \in E(\kappa, C)\right\} \cup\left\{\left([\operatorname{exit}(e)], i d_{Z_{e}}, \lambda(\mathbf{Y})\right) \mid e \in\right.$ $E(\neg \kappa, C)\}$;

$-\mathfrak{T D}=\left\{\left[s_{1}\right],\left[s_{2}\right], \operatorname{guard}(e) \wedge \operatorname{dom}[\mathbf{X} / \mathbf{X}+\mathbf{k}] \wedge Z_{e} \geq 1, \mathbf{X}^{\prime}=\mathbf{X}+\mathbf{k} \wedge\right.$ update_statevars $(\mathbf{P}) \wedge$ $\left.\left(\bigwedge_{e \in E(C)} Z_{e}^{\prime}=0\right) \mid e=\left(s_{1}, s_{2}\right) \in E(\neg \kappa, C)\right\}$;

- init $=i n i t_{0}^{+} \wedge\left(\bigwedge_{e \in E(C)} Z_{e}=0\right)$.

The previous definition gives a way to associate a TDHA to a sequential agent of a sCCP program. In order to define the TDHA for the whole program, we simply need to combine the TDHA for its components using the product construction (cf. Def. 3).

10 The stoichiometric vectors of all other continuous transitions need to be updated consequently. This will be done considering the local interaction matrix with respect to the extended set of variables. 
Definition 9. Let $\mathcal{A}=\left(A, \mathcal{D}, \mathbf{X}\right.$, dom, init $\left.t_{0}\right)$ be a simple $\boldsymbol{s} \boldsymbol{C C P}$ program and $\mathcal{A}^{+}=\left(A^{+}, \mathcal{D}^{+}, \mathbf{Y}\right.$, dom $^{+}$, init $\left._{0}^{+}\right)$ be its extended version, with $A^{+}=C_{1}\|\ldots\| C_{n}$. Fix a boolean vector $\kappa_{i}$ for each sequential agent $C_{i}$. The Transition-Driven Hybrid Automaton for the $\boldsymbol{s} \boldsymbol{C C P}$ program $\mathcal{A}$, w.r.t. $\kappa=\left(\kappa_{i}\right)_{i=1, \ldots n}$ is

$$
\mathcal{T}(A, \kappa)=\mathcal{T}\left(C_{1}, \kappa_{1}\right) \otimes \cdots \otimes \mathcal{T}\left(C_{n}, \kappa_{n}\right) .
$$

\subsection{Lattice of TDHA}

Definition 9 associates a TDHA to a $\mathbf{~ C C P}$ agent for a fixed partitioning of transitions into discrete and continuous, given by the vector $\kappa$. Clearly, different choices of $\kappa$ correspond to different TDHA's, with a different degree of approximation w.r.t. the original sCCP program. The different TDHA's that can be associated to a program $\mathcal{A}$ can be arranged into a lattice, according to the following ordering:

Definition 10. Let $\mathcal{A}$ be a sCCP agent, then $\mathcal{T}\left(\mathcal{A}, \kappa_{1}\right) \sqsubseteq \mathcal{T}\left(\mathcal{A}, \kappa_{2}\right)$ iff $\kappa_{1}[e]=1 \Rightarrow \kappa_{2}[e]=1$, for each transition $e \in E(A)=E\left(C_{1}\right) \cup \ldots \cup E\left(C_{n}\right)$, with $A=C_{1}\|\ldots\| C_{n}$ the initial agent of $\mathcal{A}$.

The bottom element of this lattice is obtained for $\kappa \equiv 0$, while the top element is obtained for $\kappa[e]=1$ iff $e$ is continuously approximable. ${ }^{11}$ These two choices correspond to two particular TDHA, as shown in the following propositions.

Proposition 2. Let $\mathcal{A}$ be a sCCP program. The TDHA $\mathcal{T}(\mathcal{A}, 0)$ is equivalent to a timed-automata with skewed clock variables $Z_{e}, e \in E\left(A^{+}\right)$, and with system and state variables modified by resets only.

Proof. The property holds for the TDHA associated to a sequential component $C$ because:

1. all transitions of $\mathcal{T}(C, 0)$ are discrete;

2. all state variables $\mathbf{P}$ and system variables $\mathbf{X}$ are governed by the ODE's $\dot{X}=0$, hence they can be modified only by resets of discrete transitions;

3. between two discrete transitions, the value of variables $\mathbf{X}$ and $\mathbf{P}$ is constant, hence the ODE's for $Z_{e}$ are of the form $\dot{Z}_{e}=$ const and each $Z_{e}$ is a skewed clock.

The property is clearly preserved by TDHA product.

Proposition 3. Let $\mathcal{A}$ be a $\boldsymbol{s} \boldsymbol{C C P}$ program with initial agent $A=C_{1}\|\ldots\| C_{n}$. If $e$ is continuously approximable for each $e \in E(A)=E\left(C_{1}\right) \cup \ldots \cup E\left(C_{n}\right)$, then $\mathcal{H}_{\mathcal{T}(\mathcal{A}, \mathbf{1})}$, the hybrid automaton derived from $\mathcal{T}(\mathcal{A}, \mathbf{1})$ coincides with the system of ODE's associated to $\mathcal{A}$ by its fluid-flow approximation.

Proof. $\mathcal{T}(A, \mathbf{1})$ has one single mode and no discrete edges, hence all transition are continuous. In this case, it is easy to see that, for each component $C$, flow $C[s]=\Phi_{C}$ and flow $_{C_{1} \| C_{2}}[s]=$ flow $_{C_{1}}[s]+$ flow $_{C_{2}}[s]=\Phi_{C_{1}}+\Phi_{C_{2}}=\Phi_{C_{1} \| C_{2}}$.

\section{Conclusion and Further Directions}

In this work we presented a method to map a given sCCP program to a lattice of Hybrid Automata, partially ordered by the level of discreteness maintained. The natural question to ask at this point if and how a sensible level of discreteness can be (semi) automatically determined, in order to maximize both expressiveness and efficiency. In our opinion this issue could be addressed syntactically by working on classes of formulae expressing significant properties for which a maximum level of discreteness can be maintained without loosing satisfiability.

Another line of work, related with expressiveness/efficiency ratio, consist in designing a dynamic version of the technique proposed here, by turning the vector $\kappa$ introduced in Section 4 into a function dependent on parameters chosen by the user, whose value can change at run-time.

Finally, we observe that the overall technique starts from the assumption that the stochastic ingredient should be eventually fully eliminated. It is easy to argue that there are situations in which this is not a good policy and, therefore, it would be interesting to study a variant of the method presented here capable to map on stochastic Hybrid Automata.

\footnotetext{
${ }^{11}$ We remind to the reader that transitions not continuously approximable must be kept discrete.
} 


\section{Bibliography}

[Bor06] L. Bortolussi. Stochastic concurrent constraint programming. In Proceedings of 4th International Workshop on Quantitative Aspects of Programming Languages (QAPL 2006), volume 164 of ENTCS, pages 65-80, 2006.

[BP07] L. Bortolussi and A. Policriti. Stochastic concurrent constraint programming and differential equations. In Proceedings of Fifth Workshop on Quantitative Aspects of Programming Languages, QAPL 2007, ENTCS 16713, 2007.

[BP08a] L. Bortolussi and A. Policriti. Hybrid approximation of stochastic concurrent constraint programming. In Proceedings of IFAC 2008. Seoul, 2008.

[BP08b] L. Bortolussi and A. Policriti. The importance of being (a little bit) discrete. In Proceedings of FBTC'08, ENTCS17346, 2008.

[BP08c] L. Bortolussi and A. Policriti. Modeling biological systems in concurrent constraint programming. Constraints, 13(1), 2008.

[DW98] A. Di Pierro and H. Wiklicky. An operational semantics for probabilistic concurrent constraint programming. In Proceedings of IEEE Computer Society International Conference on Computer Languages, 1998.

[GJS97] V. Gupta, R. Jagadeesan, and V.A. Saraswat. Probabilistic Concurrent Constraint Programming. In Proceedings of CONCUR'97, 1997.

[GJP99] V. Gupta, R. Jagadeesan, and P. Panangaden. Stochastic processes as concurrent constraint programs. In Proceedings of POPL'99, 1999.

[Hen96] T. A. Henzinger. The theory of hybrid automata. In LICS '96: Proceedings of the 11th Annual IEEE Symposium on Logic in Computer Science, 1996.

[Nor97] J. R. Norris. Markov Chains. Cambridge University Press, 1997.

[Ros96] S. M. Ross. Stochastic Processes. Wiley, New York, 1996.

[Sar93] V. A. Saraswat. Concurrent Constraint Programming. MIT press, 1993.

\section{Appendix}

\section{A Running example}

We will illustrate the notions introduced in the paper by means of an example coming from biological systems. Specifically, we consider the model of a (procaryotic) genetic regulatory network with a single gene, expressing a protein acting, after dimerization, as a repressor of its own production. We assume a cooperative repression: two dimers can bind to the promoter region of the gene. The sCCP model is given by $\mathcal{A}=(A, \mathcal{D}, \mathbf{X}$, dom, init $)$, where the variables are $\mathbf{X}=\left\{X_{p}, X_{p 2}\right\}$, storing the quantity of the protein $p$ and of its dimer $p 2$ and the components in $\mathcal{D}$ are:

$$
\begin{aligned}
& \text { gene }_{0}:-\left[* \rightarrow X_{p}^{\prime}=X_{p}+1\right]_{k_{p 1}} \text {. } \text { gene }_{0}+\left[X_{p 2}>0 \rightarrow *\right]_{k_{p 1} X_{p 2}} \text {. gene } 1 \\
& \text { gene }_{1}:-\left[* \rightarrow X_{p}^{\prime}=X_{p}+1\right]_{k_{p 2}} \text {.gene } 1+\left[X_{p 2}>0 \rightarrow *\right]_{k_{p 2} X_{p 2}} \text {. } \text { gene }_{2}+[* \rightarrow *]_{k_{u 1}} \text {. } \text { gene }_{0} \text {; } \\
& \text { gene }_{2}:-[* \rightarrow *]_{k_{u 2}} \text {.gene } 1 ; \text { deg :- }\left[* \rightarrow X_{p}^{\prime}=X_{p}-1\right]_{k_{d} X_{p}} \text {.deg; } \\
& \text { dimer }:=\left[* \rightarrow X_{p}^{\prime}=X_{p}-2 \wedge X_{p 2}^{\prime}=X_{p 2}+1\right]_{k_{x} X_{p}\left(X_{p}-1\right) / 2} \text {.dimer }+ \\
& {\left[* \rightarrow X_{p}^{\prime}=X_{p}+2 \wedge X_{p 2}^{\prime}=X_{p 2}-1\right]_{k_{-x} X_{p 2}} \text {.dimer }}
\end{aligned}
$$

Moreover, the initial network $A$ is: gene ${ }_{0}\|\operatorname{deg}\|$ dimer. The domains of variables are specified by the formula $\operatorname{dom}\left(X_{p}, X_{p 2}\right)=\left(X_{P} \geq 0\right) \wedge\left(X_{P 2} \geq 0\right)$, and the initial values of the store variables are given by $\operatorname{init}\left(X_{p}, X_{p 2}\right)=\left(X_{p}=0\right) \wedge\left(X_{p 2}=0\right)$.

The RTS of the gene component, shown in Figure 2, has three states, corresponding to the three components gene $_{0}$, gene ${ }_{1}$, and gene ${ }_{2}$, whose state variables will be denoted by $P_{0}, P_{1}$, and $P_{2}$, respectively. The RTS has also 6 transitions: $e_{1}=\left(\right.$ gene $_{0}$, gene $\left._{0}\right), e_{2}=\left(\right.$ gene $_{0}$, gene $\left._{1}\right), e_{3}=\left(\right.$ gene $_{1}$, gene $\left._{0}\right)$, $e_{4}=\left(\right.$ gene $_{1}$, gene $\left._{1}\right), e_{5}=\left(\right.$ gene $_{1}$, gene $\left._{2}\right)$, and $e_{6}=\left(\right.$ gene $_{2}$, gene $\left._{1}\right)$. 


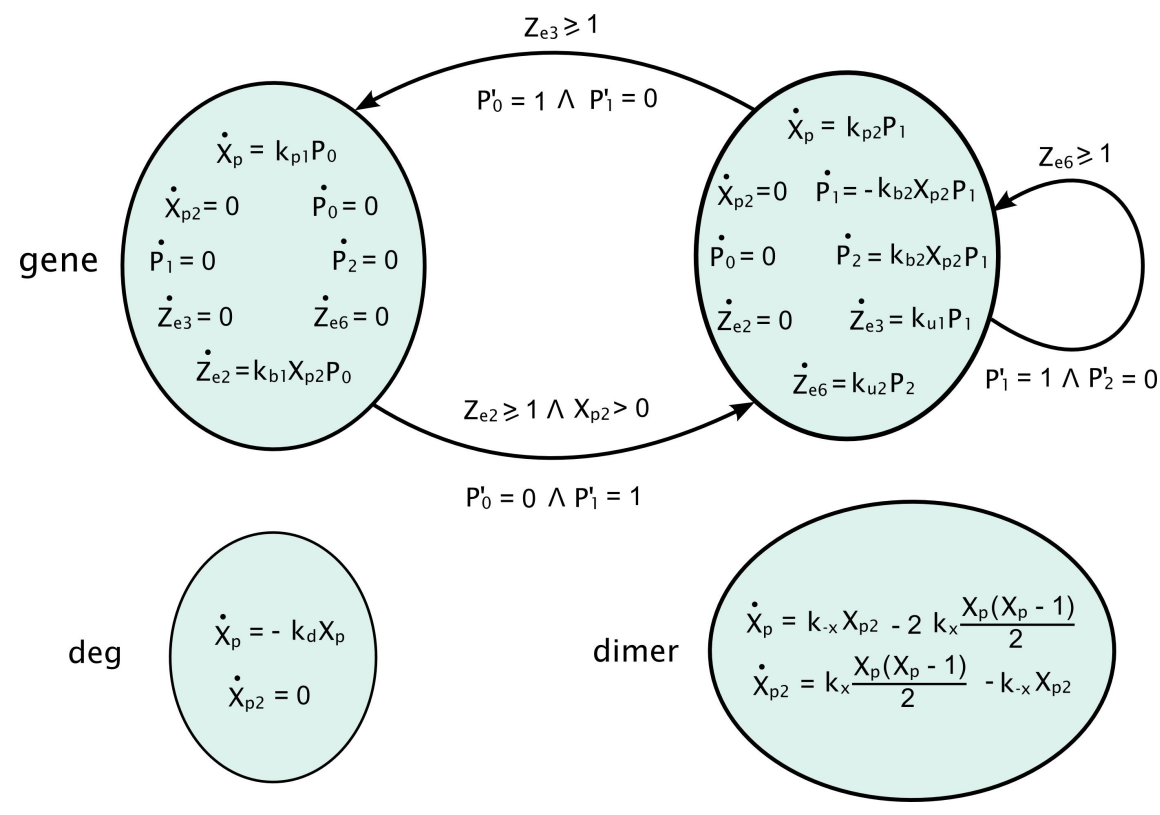

Fig. 1. Hybrid Automaton obtained from the TDHA associated to the three separate components of the $\mathbf{s C C P}$ program of Appendix A: gene, deg, and dimer. The continuous approximation scheme maintains as discrete just transitions $e_{2}, e_{3}$, and $e_{6}$ of the gene component (see the text), while all other transitions are approximated as continuous.

As an example of the effect of choosing the fixing of discreteness in the mapping to HA, we consider the $\kappa$ vector equal to $(1,0,0,1,1,0)$, which approximates edges $e_{1}, e_{4}, e_{5}$ as continuous and maintains the other three discrete. The relation $\sim_{\kappa}$ has a quotient state space containing two classes: $S_{1}=\left\{\mathrm{gene}_{0}\right\}$ and $S_{2}=\left\{\right.$ gene $_{1}$, gene $e_{2}$. The local interaction matrix and the local rate vector within state $S_{2}$ are shown below. The two columns of the interaction matrix correspond to edges $e_{4}$ and $e_{5}$, respectively.

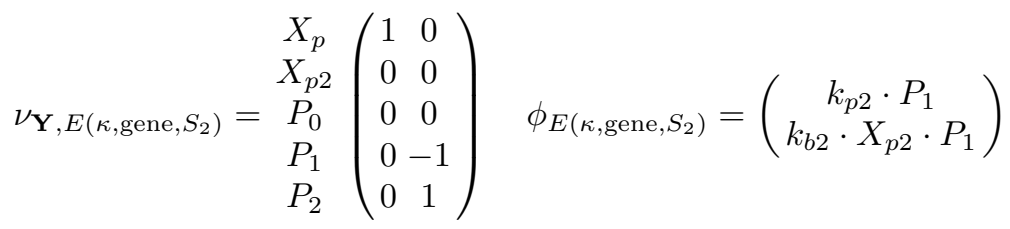

The set of continuous transitions for the TDHA associated to the gene component is: $\mathfrak{T C}=$ $\left\{\left(\left[\right.\right.\right.$ gene $\left.\left._{0}\right],(1,0,0,0,0)^{T}, k_{p 1} \cdot P_{0}\right),\left(\left[\right.\right.$ gene $\left.\left._{1}\right],(1,0,0,0,0)^{T}, k_{p 2} \cdot P_{1}\right),\left(\left[\right.\right.$ gene $\left.\left.\left._{1}\right],(0,0,0,-1,1)^{T}, k_{b 2} \cdot X_{p 2} \cdot P_{1}\right)\right\}$

The other two components, deg and dimer, have 1 , and 2 edges respectively. We consider three vectors $\kappa_{1}=(1,0,0,1,1,0), \kappa_{2}=(1)$, and $\kappa_{3}=(1,1)$. The product TDHA of these three components generates a simple HA with two states (not shown) whose discrete transition set will contain three edges, associated to RTS edges $e_{2}, e_{3}$, and $e_{6}$. Let's consider first the edge $e_{6}$. The corresponding $\mathbf{s C C P}$ transition has no guards and no updates. The TDHA edge, therefore, will be guarded by $Z_{e_{6}} \geq 1$, where the edge variable $Z_{e_{6}}$ evolves according to the differential equation $\dot{Z}_{e_{6}}=k_{u 2} P_{2}$. The update associated to this edge, according to the previous prescriptions, is $P_{2}^{\prime}=0 \wedge P_{1}^{\prime}=1 \wedge Z_{e_{2}}^{\prime}=0 \wedge Z_{e_{3}}^{\prime}=$ $0 \wedge Z_{e_{6}}^{\prime}=0$. Similarly, the discrete transition associated to edge $e_{2}$ has guard $X_{p}>0 \wedge Z_{e_{2}} \geq 1$, with $\dot{Z}_{e_{2}}=k_{b 1} X_{p 2} P_{0}$, and update $P_{0}^{\prime}=0 \wedge P_{1}^{\prime}=1 \wedge Z_{e_{2}}^{\prime}=0 \wedge Z_{e_{3}}^{\prime}=0 \wedge Z_{e_{6}}^{\prime}=0$.

Once we have the TDHA, we can generate the corresponding Hybrid Automaton, applying the construction of Definition 2. The HA associated to the TDHA for the gene component, w.r.t. $\kappa=$ $(1,0,0,1,1,0)$ is shown in Figure 1. 

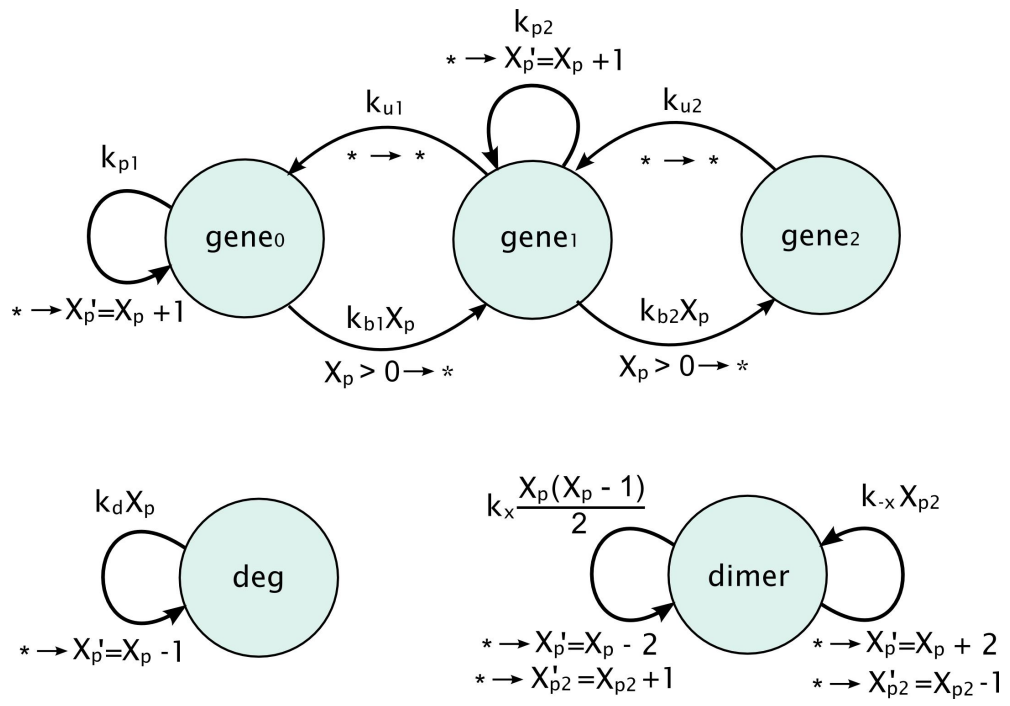

Fig. 2. Reduced Transition Systems for the three agents gene ${ }_{0}$, deg, and dimer defined in Appendix A. Each edge is labeled by its rate function and by the guard and the update (depicted in the guard $\rightarrow$ update notation).

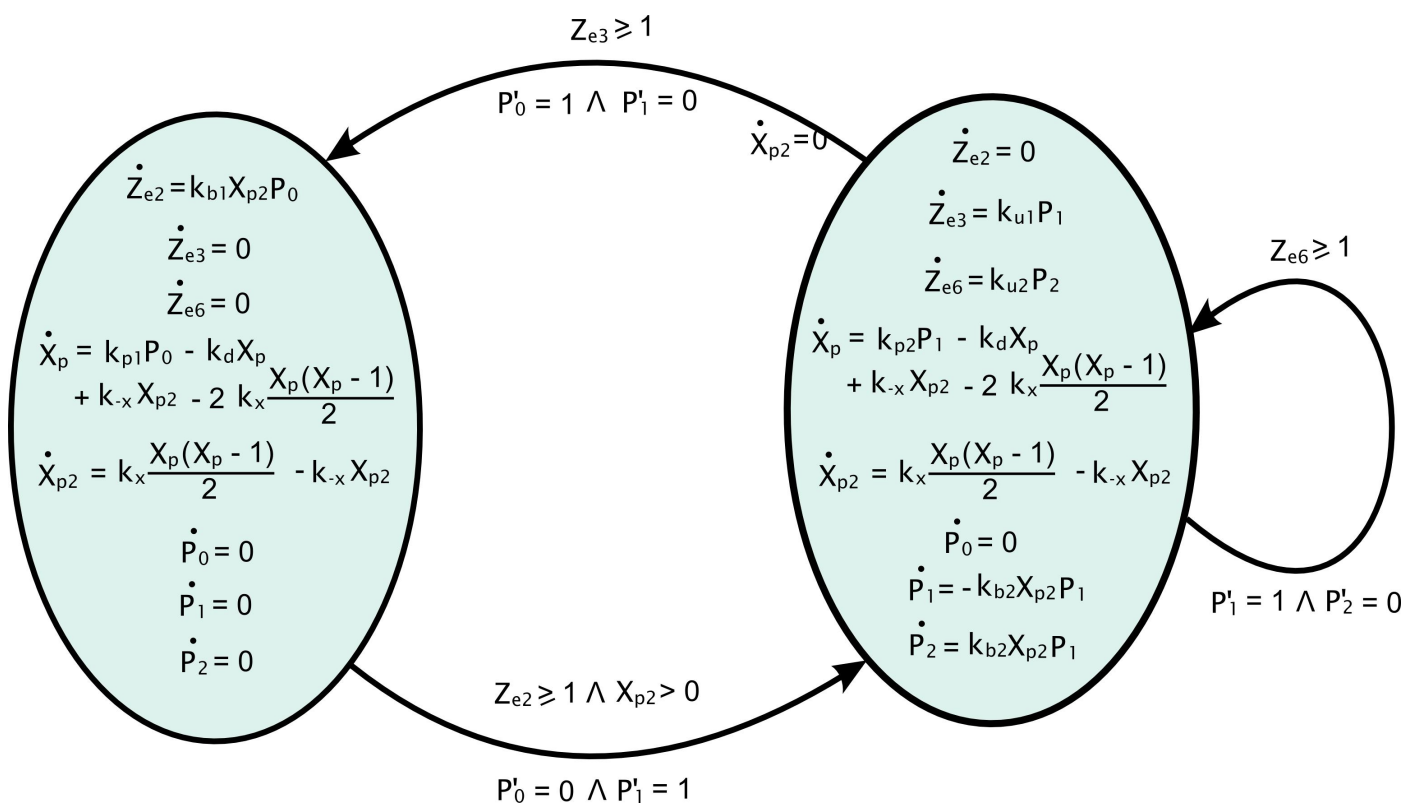

Fig. 3. Hybrid Automaton obtained from the product TDHA associated to the three components of the $\mathbf{s C C P}$ program of Appendix A, as shown in Figure 1.

\section{B Details on the LTS construction}

We give now more details on the LTS construction for sCCP. First of all, the transition relation is defined according to the rules of Table 1.

Consider the extension $\left(A^{+}, \mathcal{D}^{+}, \mathbf{Y}\right.$, dom $^{+}$, init $\left.^{+}(\mathbf{Y})\right)$ of an $\mathbf{S C C P}$ program $(A, \mathcal{D}, \mathbf{X}, \operatorname{dom}$, init $(\mathbf{X}))$, as given in Definition 5. We provide now a proof of of Proposition 1. 


$$
\begin{gathered}
((\mathbf{R} 1)) \stackrel{\mathbf{x} \vdash g(\mathbf{x}) ; \quad \mathbf{x}, \mathbf{x}^{\prime} \vdash u\left(\mathbf{x}, \mathbf{x}^{\prime}\right) ; \quad \mathbf{x}^{\prime} \vdash \operatorname{dom}\left(\mathbf{x}^{\prime}\right) ; \quad \lambda(\mathbf{x})>0}{\left\langle\left[g(\mathbf{X}) \rightarrow u\left(\mathbf{X}, \mathbf{X}^{\prime}\right)\right]_{\lambda \mathbf{x}} \cdot C+M, \mathbf{x}\right\rangle \stackrel{\lambda(\mathbf{x})}{\longrightarrow}\left\langle C, \mathbf{x}^{\prime}\right\rangle} \\
((\mathbf{R 2})) \frac{\langle M, \mathbf{x}\rangle \stackrel{\lambda}{\longrightarrow}\left\langle C^{\prime}, \mathbf{x}^{\prime}\right\rangle, C \stackrel{\text { def }}{=} M}{\langle C, \mathbf{x}\rangle \stackrel{\lambda}{\longrightarrow}\left\langle C^{\prime}, \mathbf{x}^{\prime}\right\rangle}
\end{gathered}
$$

Table 1. Structural Operational Semantics for the restricted version of sCCP presented here. The first rule deals with basic actions, the second rule with constants, and the third with parallel composition. The labels in the transition relation represents the rate of the transition. Note also how basic actions are active only if several conditions are satisfied simultaneously: the guard of the transition must be true in the current state of the store, the rate of the transition must be strictly positive and, finally, the system must not violate the domain constraints after the update.

Proposition. Let $\mathcal{A}$ be a simple sCCP program and $\mathcal{A}^{+}$be its extension. The LTS of $\mathcal{A}$ and $\mathcal{A}^{+}$are isomorphic as labeled multi-graphs.

Proof. Consider an $\mathcal{A}^{+}$-configuration $\left\langle A^{+},(\mathbf{x}, \mathbf{p})\right\rangle \in \mathfrak{A} \times \mathbb{R}^{n+m}$. We call it well-defined iff $p_{C}=$ $\#\left(C, A^{+}\right)$for $C \in \mathcal{D}^{+}$. We define now the two mappings involved in the isomorphism. Let $f(\langle A, \mathbf{x}\rangle)=$ $\left\langle A^{+},(\mathbf{x}, \mathbf{p})\right\rangle$, with $p_{C}=\#\left(C, A^{+}\right)$for $C \in \mathcal{D}^{+}$, and $g\left(\left\langle A^{+},(\mathbf{x}, \mathbf{p})\right\rangle\right)=\langle A, \mathbf{x}\rangle$. It is easy to see that $f$ and $g$ for a bijection between $\mathcal{A}$-states and well-defined $\mathcal{A}^{+}$-states.

We show now that action $\pi$ is executable in state $\langle A, \mathbf{x}\rangle$ with rate $\lambda$ iff action $\pi^{+}$is executable in state $f(\langle A, \mathbf{x}\rangle)$ with the same rate $\lambda$. Let $\pi$ be an action executable in $\langle A, \mathbf{x}\rangle$, say $\pi \in \operatorname{action}(C)$, then $C$ must be one if the agents in parallel in $A$, hence $p_{C}=\#(C, A)=1$ as $\mathcal{A}$ is simple. Now, if preconditions of guards and updates of the rule of Table 1 are satisfied by $\pi$, then so are for $\pi^{+}$(as straightforwardly checked), and rate $\left[\pi^{+}\right](\mathbf{x}, \mathbf{p})=p_{C}$ rate $[\pi](\mathbf{x})=\operatorname{rate}[\pi](\mathbf{x})$. The reverse direction from $\pi^{+}$to $\pi$ follows analogously.

We conclude the appendix giving the formal definition of reduced transition systems.

Definition 11. Let $\mathcal{A}=(A, \mathcal{D}, \mathbf{X}$, dom, init $(\mathbf{X}))$ be a $s \boldsymbol{C C P}$ program and let $C \in \mathcal{D}, C \stackrel{\text { def }}{=} M=$ $\sum_{i=1}^{k} \pi_{i} . C_{i}$, be a sequential agent.

1. The derivative set $\operatorname{Der}(C)$ of $C$ is defined recursively as $\operatorname{Der}(C)=\{C\} \cup \bigcup_{i} \operatorname{Der}\left(C_{i}\right)$.

2. The action set action $(C)$ of $C$ is action $(C)=\left\{\pi_{1}, \ldots, \pi_{k}\right\}$. The definition can be extended to sets of agents $S=\left\{C_{1}, \ldots, C_{n}\right\}$ by letting action $(S)=\bigcup_{i=1}^{n}$ action $\left(C_{i}\right)$.

In the example of Appendix A, we have, for instance, $\operatorname{Der}\left(\right.$ gene $\left._{0}\right)=\left\{\right.$ gene $_{0}$, gene $_{1}$, gene $\left._{2}\right\}$ and action (dimer $)=\left\{\pi_{1}=\left[* \rightarrow X_{p}^{\prime}=X_{p}-2 \wedge X_{p 2}^{\prime}=X_{p 2}+1\right]_{k_{x} X_{p}\left(X_{p}-1\right) / 2}, \pi_{2}=\left[* \rightarrow X_{p}^{\prime}=X_{p}+2 \wedge X_{p 2}^{\prime}=\right.\right.$ $\left.\left.X_{p 2}-1\right]_{k_{-x} X_{p 2}}\right\}$.

In the previous definition, we consider each action as a distinct object, hence two sets action $(C)$ and $\operatorname{action}\left(C^{\prime}\right)$ are always disjoint. If $C \stackrel{\text { def }}{=} \pi \cdot C^{\prime}+M$, then $\operatorname{exit}(\pi)=C$ and enter $(\pi)=C^{\prime}$, while $\operatorname{guard}(\pi)$, update $(\pi)$, and $\operatorname{rate}(\pi)$ denote respectively the guard, update and rate function of the action $\pi$. A special class of $\mathbf{~ C C P}$ agents is the following

Definition 12. Let $\mathcal{A}=(A, \mathcal{D}, \mathbf{X}$, dom, init $(\mathbf{X}))$ be a sCCP program. $\mathcal{A}$ is simple iff for each agents $C_{i}$ and $C_{j}$ in parallel in $A, \operatorname{Der}\left(C_{i}\right) \cap \operatorname{Der}\left(C_{j}\right)=\emptyset$ (i.e., there are no two copies of the same agent in A).

We can now define the Reduced Transition System:

Definition 13. Let $\mathcal{A}=(A, \mathcal{D}, \mathbf{X}, \operatorname{dom}$, init $(\mathbf{X}))$ be a $\boldsymbol{s} \boldsymbol{C C P}$ program. The reduced transition system for an agent $C \in \mathcal{D}$ is a labeled multigraph $R T S(C)=(S(C), E(C), \ell)$ defined by: 
1. $S(C)=\operatorname{Der}(C)$;

2. $E(C)=\{(\operatorname{exit}(\pi)$, enter $(\pi)) \mid \pi \in \operatorname{action}(C)\}$;

3. $\ell(e)=(\operatorname{guard}(\pi)$, update $(\pi)$, rate $(\pi))$, where $\pi$ is the action defining the edge $e \in E(C)$.

Remark 2. The syntactic restrictions introduced for sCCP seem rather strong. Indeed, the limitations on the use of parallel operator and even the requirement of being simple are only apparent restrictions. In fact, every sCCP program with agents containing parallel operators and possibly present in several copies can be transformed in an "equivalent" simple $\mathbf{s C C P}$ program, where equivalent means that the resulting CTMC is the same. 